\title{
The Effect of the Internal Field on the IR Absorption Spectra of Small Particles in Case of 3D, 2D and 1D Size Confinement
}

Igor I. Shaganov

Vavilov State Optical Institute, St. Petersburgh

Tatiana S. Perova

Trinity College Dublin

R. Alan Moore

Trinity College Dublin

See next page for additional authors

Follow this and additional works at: https://arrow.tudublin.ie/engscheceart

Part of the Electrical and Electronics Commons

\section{Recommended Citation}

Shagnov, I., Perova, T.S., Moore, R.A., Berwick, K.:Effect of the Internal Field on the IR Absorption Spectra of Small Particles in the Case of 3D, 2D, and 1D Size Confinement. J. Phys. Chem. B, 2005, 109 (20), pp 9885-9891 DOI: 10.1021/jp040543I

This Article is brought to you for free and open access by the School of Electrical and Electronic Engineering at ARROW@TU Dublin. It has been accepted for inclusion in Articles by an authorized administrator of ARROW@TU

Dublin. For more information, please contact

arrow.admin@tudublin.ie, aisling.coyne@tudublin.ie, gerard.connolly@tudublin.ie.

Funder: HEA

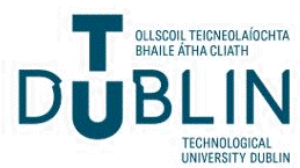




\section{Authors}

Igor I. Shaganov, Tatiana S. Perova, R. Alan Moore, and Kevin Berwick 


\title{
The effect of the internal field on the IR absorption spectra of small
}

\section{particles in case of 3D, 2D and 1D size confinement}

\author{
Igor I. Shaganov ${ }^{1}$, Tatiana S. Perova ${ }^{2 *}$, R. Alan Moore ${ }^{2}$ and Kevin Berwick ${ }^{3}$ \\ ${ }^{1}$ Vavilov State Optical Institute, 199034, St.-Petersburg, Russia \\ ${ }^{2}$ Department of Electronic and Electrical Engineering, Trinity College, Dublin 2, Ireland \\ ${ }^{3}$ Department of Electronic and Communications Engineering, Dublin Institute of \\ Technology, Kevin St, Dublin 8, Ireland
}

\begin{abstract}
The spectral properties of composite materials based on small particles with 1D, 2D and 3D size confinement are described using the combination of dispersive internal field and effective media theories approaches. The calculations performed for a number of crystalline materials have shown that the peak position and intensity of the vibrational band of the material under conditions of $1 \mathrm{D}, 2 \mathrm{D}$ and 3D size confinement are changed while the bandwidth of band remains the same. In the case of $3 \mathrm{D}$ confinement the peak position of the spectrum of isolated “mesoparticles" $\left(\varepsilon_{2}^{\text {meso }}\right)$ appears to be very close to the intrinsic frequency of the lattice vibrations, calculated from the elastic constants of this crystal, as well as to the Fröhlich's frequency. The largest shift $(\Delta v)$ of the peak frequency, $v_{\max }$, from the bulk value is obtained in the case of 1D confinement when the peak position is practically coincident with the frequency of the longitudinal optical phonon $\left(v_{\mathrm{LO}}\right)$. The aforementioned shifts are the result of intermolecular interactions, including both resonant and induced resonant dipole-dipole interactions.
\end{abstract}

\footnotetext{
*Corresponding author: email: perovat@tcd.ie; Telephone: +353-1-6081432; FAX: $+353-1-6772442$
} 


\section{Introduction}

The theory of the internal effective field dispersion of the electromagnetic wave $E_{\text {eff }}(v)$, as a general property of this field in the vicinity of the optical resonance of condensed matter, was formulated for the first time by Backshiev and co-workers. ${ }^{1,2}$ A similar approach for calculating the spectral dependence of the microscopic susceptibility in the wavelength range of the intermolecular vibrations of organic liquids was used by Clifford and Crawford. ${ }^{3}$ Analysis of experimental data using these models have shown that taking account of the internal field dispersion is useful when attempting to model the influence of intermolecular interactions (IMI) on the absorption spectra of various types of condensed matter viz. liquids, solutions and both amorphous and crystalline solids. ${ }^{1-5}$

From Refs. ${ }^{1,2}$, the relationship between the micro- and macro-characteristics of condensed matter can be presented as

$$
B(v)=\frac{n(v) K(v)}{N h \tilde{v}} \theta(v)
$$

where $B(v)$ is the spectral density of the quantum transition probability (the spectrum of the Einstein coefficient $\left.{ }^{6}\right), n(v)$ is the refractive index, $K(v)$ is the Bugger absorption coefficient, $N$ is the number of molecules (oscillators) per unit volume, $v$ is the wave number, $\theta(v)=1 /|\hat{q}|^{2}$ is the correction factor accounting for the spectral difference between the effective micro $E_{\text {eff }}(v)$ and the average macro $E(v)$ fields of the electromagnetic wave in condensed matter, and $\hat{q}=E_{\text {eff }}(v) / E(v)$ is the effective field factor, a tensor quantity in the general case. The basis for assigning the intensity of local field, which plays the role of an electro-dynamic equivalent to the combined action on the molecule of the external perturbing field and the intermolecular force, becomes the most important theoretical question in such an approach. A rigorous calculation of the local field has, to date, remained one of the most complicated problems of modern physics. 
Within the framework of the point-dipole approximation, the problem of finding the local field in homogeneous medium can be solved using a Lorentz model.

According to the Lorentz model, the effective field factor $\hat{q}$ for un-polar isotropic condensed media and for two atomic cubic crystals is given by $\hat{q}(v)=\frac{\hat{\varepsilon}(v)+2}{3}=\frac{E_{\text {eff }}(v)}{E(v)}$

where $\hat{\varepsilon}(v)=\varepsilon_{1}(v)-i \varepsilon_{2}(v)$ is the complex dielectric permittivity. It is worth noting that the external probing electric field $\mathrm{E}(\mathrm{v})$ is considered to be a weak perturbation force and therefore this approach is not applicable beyond molecular linear optics. As $K(v)=4 \pi \mathrm{k} v$, and $k=\varepsilon_{2}(v) / 2 \pi$, Eqn. (1) can be rewritten in the following form

$$
B(v)=\frac{2 \pi \varepsilon_{2}(v) \theta(v)}{N h}
$$

or alternatively as $\quad \frac{N h}{2 \pi} B(v)=\varepsilon_{2}(v) \theta(v)$

It can be shown easily that the expression on the right in Eqn. (3a) corresponds to the spectrum of the imaginary part of the complex micro susceptibility, $\hat{\chi}_{\text {mic }}(v)$, which is connected to the macroscopic susceptibility by the expression ${ }^{7}$

$$
\hat{\chi}_{\text {mic }}=\frac{3 \hat{\chi}}{\hat{\chi}+3}
$$

where $\hat{\chi}(v)=\chi_{1}(v)-i \chi_{2}(v)$. Solving Eqn. (4) with respect to $\operatorname{Im} \chi_{\text {mic }}$ we obtain $\chi_{2}^{\text {micro }}=\chi_{2} \theta(v)$. Because $\chi_{2}=\varepsilon_{2}$, the spectrum of $\chi_{2}^{\text {micro }}$ can be considered as the spectrum $\varepsilon_{2}^{\text {micro }}$. This allows to conclude that Eqn (3a) corresponds to the spectral 
characteristic of an isotropic micro-object of size $d$, satisfying the condition $\lambda>>d>>a_{\text {molec }}$, where $a_{\text {molec }}$ is the size of the molecule. So, Eqn. (3a) can be rewritten in the form

$$
\varepsilon_{2}^{\text {micro }}(v)=\varepsilon_{2}(v) \theta(v)
$$

\section{Three dimensional (3D) size confinement}

It might be supposed that Eqn. (5) allows the determination of the spectral characteristics of nanoparticles with a spheroidal shape or particles of the so called mesophase ${ }^{8}$, which satisfy the condition $d<<\lambda$, but are large enough to describe in terms of $\varepsilon(v)$. In order to confirm this assumption let us to consider the general expression for the effective dielectric permittivity function, $\hat{\varepsilon}(v)$, of a two-component composite media containing spheroidal particles of two types of isotropic materials, A an absorbing meso-phase, and B a nonabsorbing phase, in a transparent host matrix with dielectric permittivity $\varepsilon_{h}{ }^{9}$

$$
\frac{\hat{\varepsilon}^{\text {compoz. }}-\varepsilon_{h}}{\hat{\varepsilon}^{\text {compoz. }}+2 \varepsilon_{h}}=f_{A} \frac{\hat{\varepsilon}_{A}-\varepsilon_{h}}{\hat{\varepsilon}_{A}+2 \varepsilon_{h}}+f_{B} \frac{\varepsilon_{B}-\varepsilon_{h}}{\varepsilon_{B}+2 \varepsilon_{h}}
$$

$\varepsilon_{A}$ and $\varepsilon_{B}$ are the dielectric permittivity functions and $f_{A}$ and $f_{B}$ are the filling factors (or the volume concentrations) of mesophases $A$ and $B$ respectively. Eqn. (6) is a generalization of the well known Maxwell-Garnett equation, ${ }^{9,10}$ based on a LorentzLorenz model, ${ }^{9,11}$ and may be applied to spherical particles with $d<<\lambda$, but with $d>>a_{\text {molec }}$.

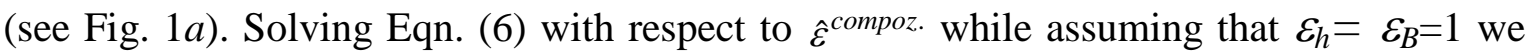
obtain

$$
\hat{\varepsilon}^{\text {compoz. }}=\frac{\hat{\varepsilon}_{A}+2+2 f_{A}\left(\hat{\varepsilon}_{A}-1\right)}{\hat{\varepsilon}_{A}+2-f_{A}\left(\hat{\varepsilon}_{A}-1\right)}
$$

By extracting the imaginary part $\left(\operatorname{Im} \hat{\varepsilon}^{\text {comp }}\right)$ from Eqn. (7) we can determine the dielectric loss spectrum of the composite media for the system under consideration 
$\operatorname{Im} \hat{\varepsilon}^{\text {comp. }}=\frac{(1+2 f) \varepsilon_{2}\left[(1-f) \varepsilon_{1}+2+f\right]-\left[(1+2 f) \varepsilon_{1}+2-2 f\right] \cdot(1-f) \varepsilon_{2}}{\left[(1-f) \varepsilon_{1}+2+f\right]^{2}+\left[(1-f) \varepsilon_{2}\right]^{2}}$

Analysis of this expression shows that the dielectric loss spectrum of the composite media at $f<<1$ is proportional to $f$ and also to the spectral micro-characteristics of phase $A$, described by Eqn. (5). For $f<<1$, expression (8) reduces to

$$
\varepsilon_{2}^{c o m p .}=\frac{9 f \varepsilon_{2}}{|\hat{\varepsilon}(v)+2|^{2}}
$$

According to Eqns. (3) and (5), this corresponds to $\varepsilon_{2}^{\text {comp }}=f \varepsilon_{2}^{\text {micro }}$. This expression shows that the spectrum of the composite media at $\varepsilon_{h}=1$ and $f<<1$ coincide, both in terms of peak position and band shape, with the spectral characteristics of the corresponding micro-particles, $\varepsilon_{2}^{\text {micro }}$, when their interactions can be neglected.

Similar expressions can be obtained from Eqn. (6) for $\varepsilon_{h}>1$ by a similar process

$$
\hat{\varepsilon}^{\text {compoz. }}=\frac{\left(\hat{\varepsilon}_{A}+2 \varepsilon_{h}\right) \varepsilon_{h}+2 f_{A}\left(\hat{\varepsilon}_{A}-\varepsilon_{h}\right) \varepsilon_{h}}{\hat{\varepsilon}_{A}+2 \varepsilon_{h}-f_{A}\left(\hat{\varepsilon}_{A}-\varepsilon_{h}\right)}
$$

By extracting the imaginary part $\operatorname{Im} \hat{\varepsilon}^{\text {comp }}$ for the case when $f<<1$ we obtain

$$
\varepsilon_{2}^{\text {comp }}=f \varepsilon_{2}^{\text {micro }}=\frac{9 f \varepsilon_{2} \varepsilon_{h}^{2}}{\left[\left(\varepsilon_{1}+2 \varepsilon_{h}\right)^{2}+\varepsilon_{2}^{2}\right]}
$$


The internal field factor in this case coincides with the expression relating the uniform field inside the spherical particle $\left(E_{i n}\right)$ with the average field $\left(E_{0}\right)$ within the medium surrounding this particle ${ }^{12}$

$$
\frac{E_{i n}}{E_{0}}=\frac{3 \varepsilon_{h}}{\left(\hat{\varepsilon}+2 \varepsilon_{h}\right)}
$$

It is worth restating at this point that $\hat{\varepsilon}(v)=\varepsilon_{1}(v)-i \varepsilon_{2}(v)$ is the complex dielectric permittivity function of the micro-particle bulk material. It can be seen from expressions (5) and (11) that the spectroscopic characteristics of the isolated particles of the mesophase do not depend solely on the dielectric properties of the corresponding bulk material but also on the dielectric properties of the matrix $\varepsilon_{h}$.

From Eqns. (9) and (11) it follows that the $\varepsilon_{2}^{\text {micro }}$ spectra physically represent the spectral characteristics of the microscopic spherical particles of the mesophase. We believe that the introduction of the $\varepsilon_{2}^{\text {micro }}$ spectra has reasonable physical grounds as these spectra can be considered as a spectrum of condensed matter under 3D size confinement. These micro-particles can be considered as "mesomolecules" possessing specific properties, thus it would be more correct to use the term $\varepsilon_{2}^{\text {meso }}$ instead of term $\varepsilon_{2}^{\text {micro }}$.

\section{Two dimensional (2D) size confinement}

In the case of 2D size confinement the condensed matter is confined in two perpendicular directions, $x$ and $y$ (Fig. 1b). This corresponds to the case of long thin rods or wires with a cross-section diameter $d$ satisfying the condition that the mesoscopic properties have been formed, i.e. for $\lambda>>d>>a_{\text {mol }}$. In order to determine the spectral characteristics of condensed matter under the conditions of 2D size confinement we need to modify the LorentzLorenz equation of the type ${ }^{8}$ 


$$
\frac{\hat{\varepsilon}-1}{\hat{\varepsilon}+1}=2 \pi N \hat{\alpha}
$$

This equation is obtained under condition that the local field tensor q is equal to

$$
\hat{q}=\frac{\hat{\varepsilon}+1}{2}
$$

According to this, the internal field correction factor $\theta(v)$, similar to the expression shown earlier for the correction factor, is described by the equation

$$
\theta_{2 D}=\frac{1}{|\hat{q}|^{2}}=\frac{4}{|\hat{\varepsilon}+1|^{2}}
$$

Therefore the spectrum of the 2D confinement micro-object, in a similar manner to Eqn. (5), can be written as

$$
\varepsilon_{2}^{\text {meso }}=\varepsilon_{2}^{\text {bulk }} \cdot \theta_{2 D}=\varepsilon_{2} \frac{4}{|\hat{\varepsilon}+1|^{2}}
$$

An example of a condensed medium consisting of this type of particle is an array of parallel wires or channels in either air or a matrix with $\varepsilon_{h}$ as shown in Fig. $1 b$. By using general expressions for composite matter Ghiner and Surdotovich, ${ }^{8}$ for instance, have obtained the following equation for the dielectric constant of wires in a vacuum

$$
\varepsilon^{c o m p}=\frac{\varepsilon+1+c(\varepsilon-1)}{\varepsilon+1-c(\varepsilon-1)}
$$

where $c$ is the concentration of wires, which correspond to the value $f$ introduced above. Note that we will use the term $f$ instead of $c$ which was used by Ghiner and Surdotovich.

Rearranging the equation (17) by analogy with formula (10) for wires in media with dielectric function of $\varepsilon_{h}$ we obtain 


$$
\hat{\varepsilon}^{\text {comp }}=\frac{\left(\hat{\varepsilon}+\varepsilon_{h}\right) \varepsilon_{h}+f\left(\hat{\varepsilon}-\varepsilon_{h}\right) \varepsilon_{h}}{\hat{\varepsilon}+\varepsilon_{h}-f\left(\hat{\varepsilon}-\varepsilon_{h}\right)}
$$

Solving Eqn.(18) for the imaginary part $\operatorname{Im} \hat{\varepsilon}^{\text {comp }}$ for strongly diluted media, we obtain

and

$$
\operatorname{Im} \hat{\varepsilon}^{\text {comp }}=f \varepsilon_{2}^{b u l k} \frac{4 \varepsilon_{h}}{\left|\hat{\varepsilon}+\varepsilon_{h}\right|^{2}}
$$

When $\varepsilon_{h}=1$ Eqn. (19) reduces to Eqn. (16)

In agreement with Eqn. (16) the Eqn. (19) can be rewritten in the form

$$
\varepsilon_{2}^{\text {comp }}=f \varepsilon_{2}^{\text {meso }}=f \varepsilon_{2}^{\text {bulk }} \theta_{2 D}
$$

These equations correspond to the orientation of the electric field vector perpendicular to the long axis of the wires, that is the case when the isotropic media is restricted to a size $<<\lambda$. So there is a screening of one medium by another which is more or less optically dense. Therefore the spectrum $\operatorname{Im} \hat{\varepsilon}^{\text {comp }}$ at $f<<1$ must be coincident in both peak position and shape to the spectrum of the appropriate 2D confined micro-object $\varepsilon_{2}^{\text {meso }}$ defined by expressions (16 and 20) where in general

$$
\theta_{2 D}=\frac{4 \varepsilon_{h}^{2}}{\left|\hat{\varepsilon}(v)+\varepsilon_{h}\right|^{2}}
$$

When the electric vector is directed along the wires in the $\mathrm{z}$ direction, i.e. there is no $2 \mathrm{D}$ confinement, the expression for the dielectric loss function is given by ${ }^{9}$

$$
\hat{\varepsilon}_{Z}^{\text {comp. }}=f_{A} \hat{\varepsilon}_{A}+f_{B} \varepsilon_{B}
$$


Due to the fact that there is no internal field correction at all in this case, the difference between the intensities of spectra of the "bulk" phase $A$ and the composite media is purely a concentration one. That is, the position and the shape of the bands coincide.

\section{One dimensional (1D) size confinement (films or pellets with thickness}

\section{$\mathrm{d}<<\lambda$, but $\left.\mathrm{d}>>\boldsymbol{a}_{m o l}\right)$}

One dimensional size confinement manifests itself in the spectral properties of super-thin films, or film structures based on it, when the electric vector of the incident light $\mathrm{E}$ is normal to the surface of these films (E॥Z on Fig. $1 c$ ). This situation occurs in Langmuir films, layers deposed on metal surfaces and monolayers of organic macro-molecules obtained under large angle impact. It can be deduced from the theory of the effective medium that in this case the normal component (EIIZ) of the dielectric loss spectrum of a stack of super-thin absorbing layers $A$, separated by transparent intermediate layers $B$, is described by the following expression ${ }^{9}$

$$
\hat{\varepsilon}_{Z}^{\text {comp }}=\left(\frac{f_{A}}{\hat{\varepsilon}_{A}}+\frac{f_{B}}{\varepsilon_{B}}\right)^{-1}
$$

If $f_{B}=1-f_{A}$ and $\varepsilon_{B}=1$, then, omitting index $A$, we obtain

$$
\hat{\varepsilon}^{\text {comp }}=\frac{\hat{\varepsilon}}{f+\hat{\varepsilon}(1-f)}
$$

and the imaginary part of the dielectric loss spectrum $\operatorname{Im} \hat{\varepsilon}_{\text {comp }}$ can be presented as follows

$$
\operatorname{Im} \hat{\varepsilon}^{\text {comp }}=\varepsilon_{2} \frac{f}{\left|\varepsilon_{1}(1-f)+f\right|^{2}+\left|\varepsilon_{2}(1-f)\right|^{2}}
$$

For $f<<1$ this equation is transformed into 


$$
\operatorname{Im} \hat{\varepsilon}^{\text {comp }}=\varepsilon_{2} \frac{f}{\left|\varepsilon_{1}\right|^{2}+\left|\varepsilon_{2}\right|^{2}}=\varepsilon_{2} \frac{f}{|\hat{\varepsilon}|^{2}}
$$

It is obvious from the Eqn. (26) that for 1D confinement, the expression for the dielectric loss spectrum for a single element of the mesophase can be presented as

$$
\varepsilon_{2}^{\text {meso }}=\varepsilon_{2}^{\text {bulk }} \theta_{1 D}=\frac{\varepsilon_{2}}{|\hat{q}|^{2}}
$$

Indeed, the internal field factor $\hat{q}$, accordingly to, ${ }^{13,14}$ is equal to $\hat{q}=\hat{\varepsilon}$ for the case of 1D confinement. Therefore, the local field correction factor appears as

$$
\theta_{1 D}=\frac{1}{|\hat{q}|^{2}}=\frac{1}{|\hat{\varepsilon}|^{2}}
$$

It follows that the dielectric loss spectrum of the composite, having a stack of $1 \mathrm{D}$ restricted layers, with $\mathrm{d}<<\lambda$ and E perpendicular to the layer surface, will be determined by the following expression

$$
\varepsilon_{2}^{\text {comp }}=f \varepsilon_{2}^{\text {bulk }} \theta_{1 D}=f \frac{\varepsilon_{2}(v)}{|\hat{\varepsilon}|^{2}}
$$

If $\varepsilon_{b}=\varepsilon_{h}>1$, then the expression for the dielectric loss spectrum of the composite medium under 1D confinement will become

$$
\varepsilon_{2}^{\text {comp }}=f \varepsilon_{2} \theta_{1 D}(v)=\boldsymbol{f}
$$


where $\mathrm{i}=1,2,3$ for $1,2,3 \mathrm{D}$ confinement accordingly. It is not difficult to show that the expression for $\theta_{i D}(v)$ is as follows

$$
\theta_{i D}(v)=\left|1+\frac{\left[\hat{\varepsilon}(v)-\varepsilon_{h}\right]}{m \varepsilon_{h}}\right|^{-2} .
$$

Here $m=1,2,3$ for cases of $1 \mathrm{D}, 2 \mathrm{D}$ and 3D confinement respectively. Note that if dipoledipole interactions between the mesophase particles are not negligible and consequently the approximation $f<<1$ is not valid, complicating the equation for the absorption spectrum of the composite media. This is because the factor $\theta_{\mathrm{iD}}(v)$ is itself becoming dependent on $\mathrm{f}$ :

$$
\theta_{i D}(v)=\left|1+\frac{(1-f)\left[\hat{\varepsilon}(v)-\varepsilon_{h}\right]}{m \varepsilon_{h}}\right|^{-2}
$$

In this case, consideration of the spectrum $\varepsilon_{2}^{\text {meso }}$ has no meaning, since as $f$ increases the absorption spectrum is gradually becoming the spectrum $\varepsilon_{2}^{\text {bulk }}$.

\section{Results and discussion}

To analyse the influence of $1 \mathrm{D}, 2 \mathrm{D}$ and $3 \mathrm{D}$ size confinement on the spectroscopic properties of condensed media in the region of the resonance absorption we used the vibrational band of a model oscillator with the following dispersion parameters: $\varepsilon_{\infty}=3$, $\rho=0.1, \gamma=0.1, \omega_{t}=1000 \mathrm{~cm}^{-1}$. The spectra of the model media in the bulk state, $\varepsilon_{2}(v)$, and under conditions of $1 \mathrm{D}, 2 \mathrm{D}$ and $3 \mathrm{D}$ size confinement, $\varepsilon_{2}^{\text {meso }}$, were calculated using the usual dispersion relationship ${ }^{15}$ as well as formulas (5), (16) and (27). The results of these calculations are shown in Fig. 2. The main parameters of the bands considered are presented in Table 1. 
The data shown in Table 1 and in Fig. 2 show that the spectral parameters are changed during the transition from the spectra of the bulk material to the spectra of this material under conditions of 1D, 2D and 3D size confinement. Note that these changes only concern the intensity and peak position of the vibrational bands, while the linewidth remains the same. This latter result is a characteristic feature of the isolated symmetrical bands. In the general case of overlapping absorption bands, a substantial redistribution of the intensities of the individual components and a consequent change in the shape of the entire spectrum is possible.

Calculations performed for a number of real materials have shown that all the aforementioned changes in the spectra of the bulk materials during the transition to the conditions of 1D, 2D and 3D size confinement are retained. Tables 2 and Fig. 3 show the spectra and their characteristics of the fundamental lattice vibration of $\mathrm{MgO}$ in the bulk state, under the conditions of size confinement and also for the composite media on it's base at $f=0.001$.

From these results it can be seen that the peak position of the absorption spectrum of MgO under $1 \mathrm{D}, 2 \mathrm{D}$ and $3 \mathrm{D}$ size confinement at $\varepsilon_{\mathrm{h}}=1$ is shifted to the high frequency side by a value of $\Delta v$ equal to 316, 252 and $209 \mathrm{~cm}^{-1}$ respectively. The largest shift, $\Delta v$, is obtained in the case of $1 \mathrm{D}$ confinement when the peak position, $v_{\max }$, is practically coincident with the frequency of the longitudinal optical (LO) phonon $v_{\mathrm{LO}}$ of the bulk MgO crystal. In the case of 3D confinement the peak position of the spectrum of the isolated mesoparticle $\left(\varepsilon_{2}^{\text {meso }}\right)$ appears to be very close to the intrinsic frequency of the lattice vibration $v_{\beta}=615$ $\mathrm{cm}^{-1}$, calculated from the elastic constants of this crystal, ${ }^{16}$ as well as to the Fröhlich's frequency $v_{\mathrm{F}}=605 \mathrm{~cm}^{-1} \cdot{ }^{17}$ These frequency shifts are the result of intermolecular 
interactions, including both resonant and induced-resonant dipole-dipole interactions. Induced-resonant dipole-dipole interactions appear as a result of the interaction of the absorbing molecule (particle) with the transparent matrix ${ }^{4}$ and reflect the influence of the electronic transition on the vibrational ones in the case of both the solutions and the homogeneous media.

In the theory describing resonant interactions, these are taken into account during the correction of the resonance shift accounting for the influence of the background polarisation. We would like to emphasise once more that the absorption spectrum in the case of 3D confinement is the analogue of the spectral density of the quantum transition probability with absorption i.e. the spectrum of the Einstein coefficient $B(v)^{6}$. It differs from it only by the numerical coefficient. It can be assumed that in this case only the spectroscopic characteristics of the mesoparticle coincide with the analogous microscopic characteristics. This statement is valid at least for isotropic spherical particles described using a Lorentz internal field model taking into account the long-range dipole-dipole interactions only. Therefore, the integral of the $\varepsilon_{2}^{\text {meso }}$ spectrum characterises the value of the square of the matrix element of the transition dipole moment, while the peak position determines the intrinsic oscillator frequency or the so called bare mechanical frequency ${ }^{18,20}$ in the absence of the external perturbation. Both the resonant and the induced-resonant dipole-dipole interactions lead to a frequency shift and intensity change of the vibrational absorption bands of the bulk materials from that for isolated molecules, microparticles or clusters. By taking into account the dispersion of the internal field the contribution of these interactions can be separated. 
If for the isolated mesoparticles we will use as a matrix a material with a dielectric permittivity $\varepsilon_{\mathrm{h}}$ equal to $\varepsilon_{\infty}$ of the bulk material of particles, then the frequency shift with respect to the peak position of the absorption spectrum of bulk material will be due to the resonant part of the intermolecular interactions potential only. The contribution of the induced-resonance interactions, that is, the background polarisation, will be determined by the difference in the frequency maximum of mesoparticles at $\varepsilon_{h}=1$ and $\varepsilon_{h}=\varepsilon_{\infty}$. In the case of $\mathrm{MgO}$ the induced-resonance part of the frequency shift will be defined as $\Delta v=610-528=$ $82 \mathrm{~cm}^{-1}$.

As can be seen from Eqns. (11), (21) and (31) the influence of $\varepsilon_{\mathrm{h}}$ on the peak position of spectral bands manifests itself only under 3D and 2D confinement. For 1D confinement (see Eqn. (31)) the deviation of the $\varepsilon_{\mathrm{h}}$ from 1 leads to an intensity change only and does not affect the peak position of the absorption band. This is because the $\varepsilon_{\mathrm{h}}$ is not included into the denominator of the equation for the local field correction factor $\theta(v)$. At the same time, an increase of $\varepsilon_{\mathrm{h}}$ will lead to an equalising of the intensity of the absorption bands for all the cases under consideration. Nevertheless, a comparison of their integrated intensities shows that even at $\varepsilon_{h}=\varepsilon_{\infty}$ the average value of the correction factor $\langle\theta(v)\rangle$ $=\int \varepsilon_{2}^{\text {meso }}(v) \mathrm{d} v / \int \varepsilon_{2}(v) \mathrm{d} v$ deviates substantially from 1 . This shows that, including the contribution from the absorption band itself, the background dielectric permittivity in the spectral region considered will be somewhat larger. It is easy to estimate that a value of $\theta=1$ corresponds to a value of $\varepsilon_{\mathrm{h}} \cong 4$. This leads to the conclusion that the contribution to the $\varepsilon_{\text {background }}$ from the band itself can be defined as $\Delta \varepsilon=4-\varepsilon_{\infty} \approx 1$. 
As was already mentioned, Eqn. (33) is only valid for the case of highly diluted composite systems where the amount of the absorbing component is small and $f<<1$. The extent of dilution will be determined by the intensity of the band in the spectrum of $\operatorname{Im} \hat{\varepsilon}(v)$ as well as by the value of $\varepsilon_{\mathrm{h}}$. As the value of $\operatorname{Im} \hat{\varepsilon}(v)$ increases, the value of $f$ at which there are no long-range resonance interactions between the separate elements of the mesoscopic phase decreases (i.e. these interactions can be neglected). In the case of MgO the results of calculations using Eqns. (33) and (34) practically coincide at $f=0.001$ (see Fig. 3). At the same time the intensities of the absorption spectra of composite media are 1000 times smaller than the consequent intensities of spectra of the isolated particles of the mesophase embedded into the same matrix.

\section{Conclusions}

In conclusion we note that the expressions obtained in the present work are in agreement with the theory of light absorption by small particles [12]. Although these expressions still describe the idealised case of ordered oriented mesoparticles of limited size in the direction of confinement and an ideal geometrical shape, these can be generalised to the case of the freely oriented particles of spheroidal shape by taking into account the ratio of hemispheric axes of corresponding spheroids. Nevertheless, the experimental data available appears to be in agreement with these expressions under certain conditions. For instance, it should be noticed that expression (27) for $\varepsilon_{2}^{\text {meso }}$ for 1D confinement coincides with the expression for the dielectric loss spectrum at the frequencies of the longitudinal optical (LO) vibrations of crystalline solids. This shows that these absorption bands will appear under 1D size confinement in any condensed media.

This conclusion is in agreement with experimental results on LO-TO spectral splitting observed in glasses and is also supported by work on the role of the dispersive internal 
field in the formation of reflection-absorption spectra of thin amorphous layers of silicon dioxide $^{18}$. A similar conclusion also follows from the theoretical work of Lechman ${ }^{19}$, who demonstrated that the appearance of absorption at the frequency of LO vibrations in structure-less dielectrics is a result of the boundary conditions arising in thin films at an oblique incidence of light. Thus, we can conclude that the case of $1 \mathrm{D}$ size confinement is apparent in the reflection-absorption spectra (RAS) of thin dielectric films independent of their structure. It was shown earlier that intense vibrational bands in RAS spectra obtained at incident angles in the range of $70^{\circ}-80^{\circ}$ are apparent at frequencies of $v \geq v_{\mathrm{LO}}$. As film thickness decreases, the peak position of these bands is practically coincident with the frequency $v_{\mathrm{LO}}$ corresponding to the ratio (27). The same results were observed for thin amorphous films of $\mathrm{MgO}, \mathrm{ZrO}_{2}$ and $\mathrm{SiO}_{2}{ }^{20,21}$.

Other experimental data, supporting the conclusions arrived in this paper, are contained in work on the absorption spectra of powder consisting of microcrystals of alkali halides. As was shown in our previous papers ${ }^{22,5}$ the frequencies of the absorption spectra for these powders in some cases appears to be very close to the frequency of $B(v)$ spectra of their bulk counterparts and therefore close to the spectra of $\varepsilon_{2}^{\text {meso }}$. Nevertheless it is obvious, that the expressions obtained in this work cannot give a universal description of the absorption spectra of small particles, since they do not account for the actual sizes of the particles as well as their shape. This fact probably accounts for the deviation between experimental results and theoretical calculations in the case of 3D size confinement observed for MgO. In accordance with Ref. ${ }^{23}$, the frequency maximum of the absorption spectra of cubic $\mathrm{MgO}$ microcrystals in air is $v_{\text {meso }}=558 \mathrm{~cm}^{-1}$ instead of the $610 \mathrm{~cm}^{-1}$ calculated in this work. We believe that this discrepancy could be attributed to 
i) deviation of the particle shape from spherical

ii) insufficiently small particle size and

iii) aggregation effects.

Therefore the relationships obtained here require further elaboration and experimental verification. We believe that the approach, based on the spectroscopy of intermolecular interactions, suggested here can be used as a general methodology for the description of nanostructures. Though the results presented here can be considered as preliminary, they show that the dispersive internal field method can be useful for the prognosis of the spectroscopic characteristics of diluted nanocomposites of various types on the basis of the establishing relations between spectral characteristic of different small particles and their bulk counterparts. Preliminary investigations show that the interactions of the separate elements of these structures can be considered as an interaction of "mesomolecules" which possess their own intrinsic properties. A detailed elaboration of these characteristics taking into account the size, shape and crystalline structure of microparticles and also with the properties of the surrounding media could be done. This would require more advanced calculations of the internal field factor taking into account the long-range and short-range intermolecular interactions.

\section{Acknowledgments:}

The authors thank the HEA, Ireland for financial support of this work. Thanks due to Daria Potapova and Saritha Unnikrishnan for help with calculations. 


\section{References:}

1. Backshiev, N. G. Spectroscopy of intermolecular interactions; Nauka: Leningrad, 1972. (In Russian).

2. Bakhshiev, N. G.; Girin, O. P.; Libov, V. S. a) Sov.Phys.Dokl. 1963, 7, 719; b) Sov. Opt. \& Spectr. 1963, 14, 476.

3. Clifford, A. A.; Crawford, B. J. Phys. Chem. 1966, 70, 1536.

4. Libov, V. S. Rus. J. Phys.Chem. 1980, 54, 817.

5. Shaganov, I. I. Sov. J, Opt. Technol. 1992, 59, 1; Rus. Opt. Spectrosc. 1980, 49, 97.

6. Heitler, W. Quantum Theory of Radiation, 3rd ed.; Wiley: New York, 1975.

7. Grosse, P. Freie electronen in Festkorpern; Springer-Verlag, Berlin, Heidelb: New York, 1979.

8. Ghiner, A. V.; Surdutovich, G. I. Phys.Rev.A, 1994,50, 714.

9. Aspnes, D. E. Am. J. Phys., 1982, 50, 704.

10. Maxwell-Garnett, J. C. Philos. Trans., 1906, A205, 2371.

11. Lorentz, A. Theory of Electrons, $2^{\text {nd }}$. ed.; Teubner: Leipzig, 1916.

12. Van de Hulst, H. C. Light Scattering by Small Particles; Dover: New York, 1981.

13. Stratton, J. A. Electromagnetic Theory; MGr. Hill: New York, 1941.

14. Ghiner, A.V.; Surdotovich, G.I. Optics \& Potonics News, 1994, 34.

15. Jaspers, I. R.; Kahan, A.; Plendle, I.N.; Mitra, I.N. Phys.Rev. 1966, 146, 526.

16. Tolstykh (Perova), T.S.; Shaganov, I.I.; Libov, V.S. Sov. Phys. Solid State, 1974, 16, 431.

17. Pigenet, C.; Fievet, F. Phys Rev.B, 1980, 22, 2785.

18. Shaganov, I. I.; Perova, T.S.; Moore, R.A.; Berwick, K. J. Mater. Sci.: Mater.Electr., 2001, 12, 351. 
19. Lehmann, A. Phys.St.Sol. (b), 1988, 148, 401.

20. Shaganov, I. I.; Perova, T.S.; Moore, R.A.; Berwick, K. SPIE, 2003, 4876, 1158.

21. Konovalova, O. P.; Rusakova, O.Yr.; Shaganov, I.I. Sov.J.Opt.Technol. 1988, 55, 402.

22. Tolstykh (Perova), T. S.; Libov, V.S. in Spectrochemistry of Intra- and Intermolecular Interactions, n.1; Leningrad State Univ.Press: Leningrad, 1975.

23. Genzel, L.; Martin, T. P. Phys. Status Solidi (b), 1972, 51, 101. 